\title{
Paraneoplastic Syndrome of Metastatic Neuroendocrine Carcinoma: Presentation of Recurrent Hyponatremia
}

\author{
Seo-Hee Yang ${ }^{1}$, Kyoung Min Kim ${ }^{2}$, and Kyung Pyo Kang ${ }^{1,3, *}$ \\ ${ }^{1}$ Department of Internal Medicine, Research Institute of Clinical Medicine, Jeonbuk National University Medical School, ${ }^{2}$ Department of \\ Pathology, Jeonbuk National University Medical School, ${ }^{3}$ Biomedical Research Institute, Jeonbuk National University Hospital, Jeonju, Korea
}

Lung neuroendocrine tumors (NETs) are a rare clinical condition of pulmonary neoplasms and are histologically characterized by neuroendocrine differentiation. ${ }^{1}$ Due to their neuroendocrine cellular origin, these tumors may produce a biologically active peptide, which results in paraneoplastic syndrome. Among them, the syndromes of inappropriate antidiuretic hormone secretion (SIADH), characterized by abnormally elevated levels of antidiuretic hormone $(\mathrm{ADH})$, lead to impairment of free water excretion and results in significant electrolyte abnormalities, such as hyponatremia. ${ }^{2}$ Here, we report a case of a recurrent symptomatic hyponatremia patient who was finally diagnosed with metastatic neuroendocrine carcinoma of the liver and pleura.
An 83-year-old woman was admitted to the hospital for evaluation of epigastric and right pleuritic chest pain. She had been diagnosed with gastric cancer at 16 years ago and had a subtotal gastrectomy. On admission, she was alert and oriented with no pretibial pitting edema. Her breathing sound was normal. Mild tenderness on epigastric area was noted. Laboratory findings revealed a serum creatinine level of $0.51 \mathrm{mg} / \mathrm{dL}$, serum sodium of $119 \mathrm{mmol} / \mathrm{L}$, glucose of $135 \mathrm{mg} / \mathrm{dL}$ and serum osmolality of $244 \mathrm{mOsm} / \mathrm{kg}$. The Serum cortisol level was $17.3 \mu \mathrm{g} / \mathrm{dL}$, and the free T4 and thyroid stimulation hormone level (TSH) was $16.0 \mathrm{pmol} / \mathrm{L}$ (reference range: $11.5-22.7 \mathrm{pmol} / \mathrm{L}$ ) and $3.33 \mu \mathrm{IU} / \mathrm{mL}$ (reference range: $0.55-4.78 \mu \mathrm{IU} / \mathrm{mL}$ ) at the early morning blood sample. Urine electrolytes were sodium of $64 \mathrm{mmol} / \mathrm{L}$
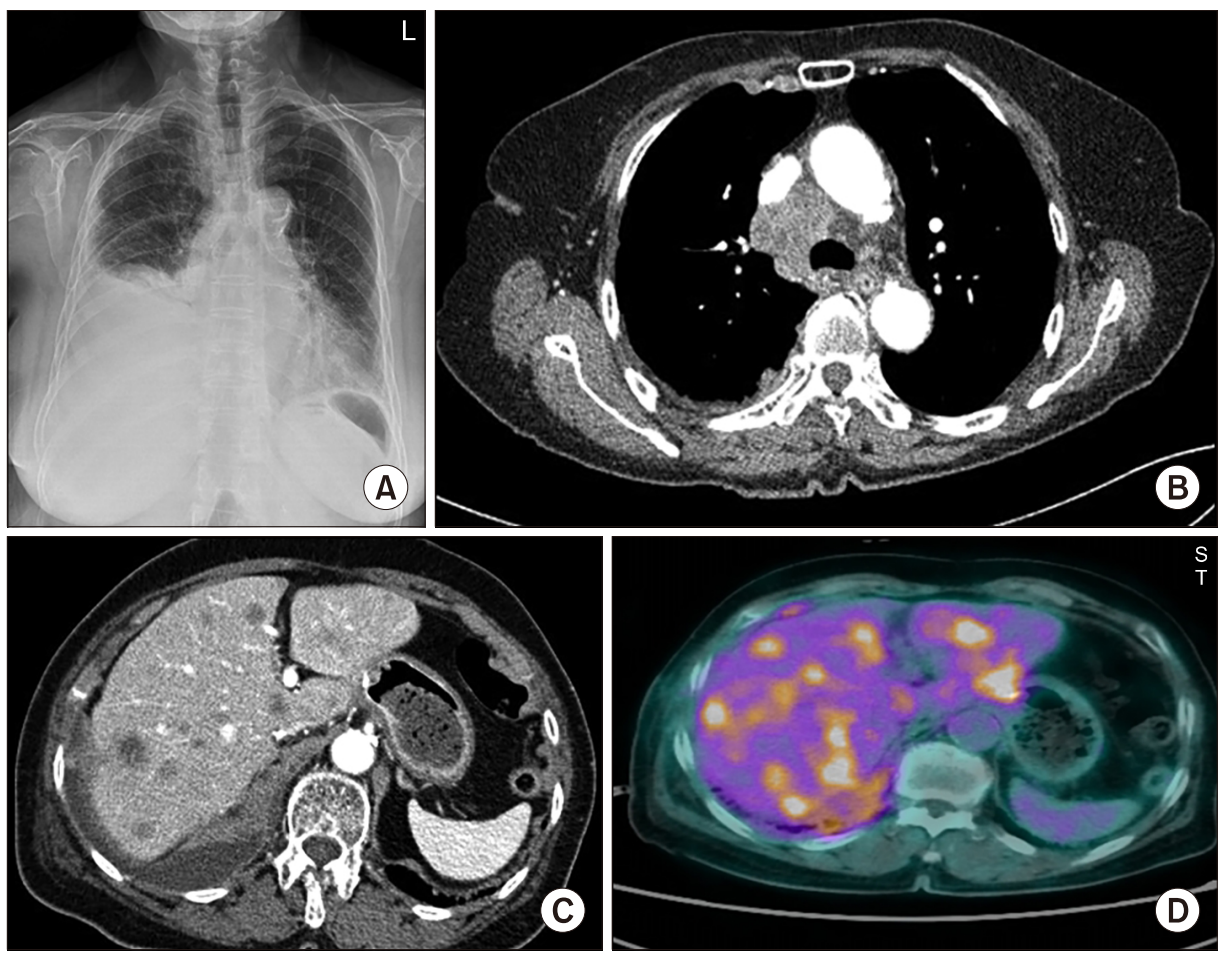

FIG. 1. Radiologic findings. (A) Chest $\mathrm{x}$-ray shows right pleural effusion. (B) Chest CT shows the multiple mediastinal lymph node metastases as well as right pleura, right internal mammary chain. (C) Abdomen CT shows the multiple enhancing nodules in the liver, suggestive of metastatic nodules. (D) PETCT imaging shows intense FDG in the liver lesions suggestive of metastasis.
Corresponding Author:

Kyung Pyo Kang

Department of Internal Medicine, Jeonbuk National University Medical School, 20 Geonji-ro, Deokjin-gu, Jeonju 54907, Korea Tel: +82-63-250-2361, Fax: +82-63-254-1609, E-mail: kpkang@chonbuk.ac.kr

\section{Article History:}

Received November 26, 2019 Revised January 24, 2020

Accepted February 13, 2020 

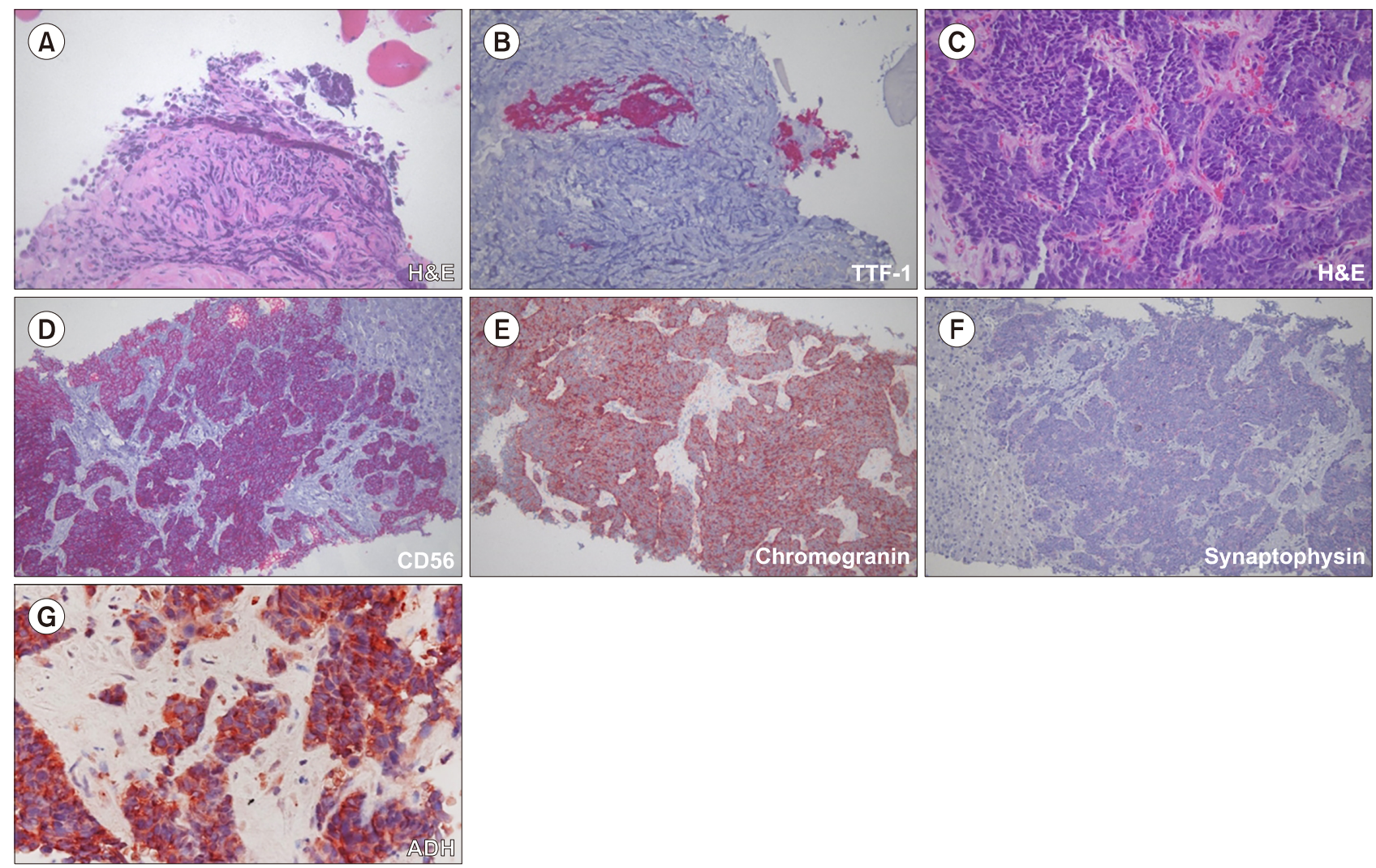

FIG. 2. Histologic findings of pleura and liver. (A, B) Pleural biopsy shows a few atypical cells (H\&E, original magnification, 400× expressing thyroid transcription factor-1 (TTF-1) between crushed inflammatory cells (original magnification, 200×). (C-F) Liver biopsy shows an organoid pattern of tumor cells (H\&E, original magnification, 400x), which are positive for CD56, chromogranin and synaptophysin (original magnification, 200×). These findings confirm neuroendocrine carcinoma. (G) Immunohistochemical staining for antidiuretic hormone $(\mathrm{ADH})$ in the tumor. The tumor cells show strong cytoplasmic expression for $\mathrm{ADH}$ (original magnification, 400x).

and urine osmolality of $421 \mathrm{mOsm} / \mathrm{kg}$. The patient's hyponatremia was resolved with fluid restriction and an infusion of hypertonic saline. Eight weeks after first admission, her euvolemic hyponatremia had recurred. There were new lesions on a chest X-ray, unilateral pleural effusion (Fig. 1A). Therefore, further evaluation was conducted to rule out the malignancy-associated SIADH. A chest computed tomography (CT) scan showed multiple mediastinal lymph node metastases as well as at the right pleura and the right internal mammary chain with heterogeneous enhancement (Fig. 1B). An abdomen CT scan showed multiple $2 \mathrm{~cm}$-sized rim enhanced metastatic nodules in the liver and no recurrent masses at operation anastomosis site (Fig. 1C). For the evaluation of malignancy, fluorine-18-2fluoro-2-deoxy-D-glucose (FDG) positron emission tomography/computed tomography (PET-CT) was implemented, and intense FDG uptake was seen in an axial fused PET-CT image of the liver lesions suggestive of metastasis (Fig. 1D). Pleural biopsy showed a few atypical cells (Fig. 2A; H\&E, 400x) with expressing thyroid transcription factor-1 (TTF-1) (Fig. 2B; 200x). Liver biopsy showed an organoid pattern of tumor cells (Fig. 2C; H\&E, 400x), which were positive for CD56, chromogranin, and synaptophysin (Fig. 2D-F; 200x). To confirm malignancy-associated SIADH, immuno- histochemical staining for ADH was conducted in a liver biopsy. There was strong cytoplasmic expression for $\mathrm{ADH}$ in tumor cells (Fig. 2G; 400x).

The patient had two cycles of etoposide and cisplatin chemotherapy for neuroendocrine tumors. However, her general condition rapidly deteriorated with recurrent hyponatremia despite the treatment by tolvaptan and the patient died after hospice care.

The presence of such syndromes is important as their clinical presentation, if not identified, may delay the diagnosis of the underlying neoplasia. Conversely, early recognition can allow for more rapid diagnosis, particularly as the coexistence of a neoplasm with a clinical or biochemical markers offers an additional determinant of tumor status and progression. We also showed a direct relationship between neuroendocrine tumors and hyponatremia by immunohistochemical stains for $\mathrm{ADH}$. This case emphasizes the importance of early recognition of SIADH, which may be the only initial manifestation of metastatic neuroendocrine carcinoma.

\section{CONFLICT OF INTEREST STATEMENT}

None declared. 


\section{REFERENCES}

1. Travis WD. Advances in neuroendocrine lung tumors. Ann Oncol 2010;21 Suppl 7:vii65-71.
2. Howard JD Jr, Deveaux PG. Syndrome of inappropriate antidiuretic hormone associated with rectal small cell neuroendocrine carcinoma: a case report. J Surg Case Rep 2017;2017:rjx136.

This is an Open Access article distributed under the terms of the Creative Commons Attribution Non-Commercial License (http://creativecommons.org/licenses/ by-nc/4.0) which permits unrestricted non-commercial use, distribution, and reproduction in any medium, provided the original work is properly cited. 